\title{
Piezoelectricity in nominally centrosymmetric phases
}

\author{
Oktay Aktas $\odot,{ }^{1, *}$ Moussa Kangama, ${ }^{1}$ Gan Linyu,,${ }^{1}$ Gustau Catalan $\odot,{ }^{2}$ Xiangdong Ding,,${ }^{1, \dagger}$ \\ Alex Zunger, ${ }^{3}$ and Ekhard K. H. Salje $\odot^{4, \sharp}$ \\ ${ }^{1}$ State Key Laboratory for Mechanical Behavior of Materials \& Materials Science and Engineering, \\ Xi'an Jiaotong University, Xi'an 710049, China \\ ${ }^{2}$ ICREA and ICN2-Institut Catala de Nanociencia i Nanotecnologia, Campus UAB, 08193 Bellaterra (Barcelona), Catalonia \\ ${ }^{3}$ University of Colorado, Energy Institute, Boulder, Colorado 80309, USA \\ ${ }^{4}$ Department of Earth Sciences, University of Cambridge, Downing Street, Cambridge CB2 3EQ, United Kingdom
}

(Received 3 May 2021; revised 2 August 2021; accepted 11 November 2021; published 27 December 2021)

\begin{abstract}
Compound phases often display properties that are symmetry forbidden relative to their nominal, average crystallographic symmetry, even if extrinsic reasons (defects, strain, or imperfections) are not apparent. Specifically, breaking the macroscopic inversion symmetry of a centrosymmetric phase can dominate or significantly change its observed properties while the detailed mechanisms and magnitudes of the deviations of symmetry breaking are often obscure. Here, we choose piezoelectricity as a tool to investigate macroscopic inversion-symmetry breaking in nominally centrosymmetric materials as a prominent example and measure resonant piezoelectric spectroscopy (RPS) and Resonant Ultrasound Spectroscopy (RUS) in 15 compounds, 18 samples, and 21 different phases, including unpoled ferroelectrics, paraelectrics, relaxors, ferroelastics, incipient ferroelectrics, and isotropic materials with low defect concentrations, i.e., $\mathrm{NaCl}$, fused silica, and $\mathrm{CaF}_{2}$. We exclude the flexoelectric effect as a source of the observed piezoelectricity yet observe piezoelectricity in all nominally cubic phases of these samples. By scaling the RPS intensities with those of RUS, we calibrate the effective piezoelectric coefficients using single-crystal quartz as standard. Using this scaling we determine the effective piezoelectric modulus in nominally nonpiezoelectric phases, finding that the "symmetry-forbidden" piezoelectric effect ranges from $\sim 1$ to $10^{-5} \mathrm{pm} / \mathrm{V}\left(\sim 0.5 \%\right.$ to $\sim 2 \times 10^{-5} \%$ of the piezoelectric coefficient of poled ferroelectric lead zirconate titanate). The values for the unpoled ferroelectric phase are only slightly higher than those in the paraelectric phase. The extremely low coefficients are well below the detection limit of conventional piezoelectric measurements and demonstrate RPS as a convenient and ultrahighly sensitive method to measure piezoelectricity. We suggest that symmetry-breaking piezoelectricity in nominally centrosymmetric materials and disordered, unpoled ferroelectrics is a common phenomenon.
\end{abstract}

DOI: 10.1103/PhysRevResearch.3.043221

\section{INTRODUCTION}

Along with chiral dichroism, second harmonic generation, and Rashba spin splitting, the classic effects of piezoelectricity and pyroelectricity belong to a group of functionalities enabled by specific crystal class (CC) symmetries. Both effects require an absence of inversion center, i.e., belonging to a noncentrosymmetric (NCS) crystal class. In addition, whereas pyroelectricity is restricted exclusively to polar crystal classes, piezoelectricity is allowed both in polar symmetries as well as in nonpolar symmetries (with the exception of the nonpolar $\mathrm{CC}$ of type $\mathrm{O}$ that forbids piezoelectricity). Remarkably, piezoelectricity and pyroelectricity were recently observed

\footnotetext{
*oktayaktas@xjtu.edu.cn

†dingxd@xjtu.edu.cn

‡ekhard@esc.cam.ac.uk
}

Published by the American Physical Society under the terms of the Creative Commons Attribution 4.0 International license. Further distribution of this work must maintain attribution to the author $(s)$ and the published article's title, journal citation, and DOI. in nominally centrosymmetric phases such as the cubic paraelectric phases of certain oxides [1-10]. Piezoelectricity was demonstrated in several well-known materials, such as paraelectric phase of $\mathrm{BaTiO}_{3}$ and its solid solutions, ferroelastic $\mathrm{LaAlO}_{3}$ and $\mathrm{SrTiO}_{3}$, and relaxor ferroelectric lead magnesium niobate (PMN) [3-5,8-12], raising much attention both in the scientific and engineering communities $[6,9,13-15]$.

Some of the sightings of piezoeffects in centrosymmetric phases are perhaps less than compelling, being likely false positive determinations of the nominal cubic phase. Here, the centrosymmetric phase may be the paraphase of a ferroelectric material, where the phase transition from the ferroelectric phase is not properly completed. Such "false positive" observations thus do not necessarily pertain to nominally cubic phases (e.g., when the measurement temperatures are just below the para-to-ferro transition point) and thus the appearance of piezoelectricity does not pose a puzzle. Other mechanisms, once thought to explain the observation of piezoeffects, will be argued below not to be plausible. For example, one needs to be careful to exclude both flexoelectricity and surface piezoelectricity as experimental errors which are common in heavily strained samples but do not lead to piezoelectricity. 
The remaining data and observations of piezoelectricity in nominal cubic materials are broadly divided into two causes.

a. Ferroelastic domains within ferroelastic phases and ferroelastic local domains in the paraphase. These are related to the ordering of spontaneous strain in ferroelastic materials, which are mechanical analogs of ferroelectrics (spontaneous polarization) and ferromagnets (spontaneous magnetization) [16-20]. In the ferroelastic phase, twin walls, i.e., boundaries separating ferroelastic domains (or strain states), were shown to be generally polar [6]. Above the transition temperature, signatures of the ferroelastic phase (also called precursors) occur as strain fluctuations (in the paraelastic phase), leading to structural heterogeneity [16-18,21]. These include tweed patterns that are seen in electron diffraction experiments as cross-hatched patterns and were observed to have lengths of 100 to $2000 \AA$ [19]. Such heterogeneities can be a result of elastic anisotropy, the interaction of local strain with another property, such as octahedral tilting in some perovskite oxides and halides, polarization in incipient ferroelectrics, or compounds whose ferroelectric or ferromagnetic phase is simultaneously ferroelastic [16,19,20,22].

b. Ferroelectriclike local polar structures within the paraelectric phase. These give rise to intrinsic piezoelectricity although some defect-induced polarity may play a role as extrinsic stimulus [16-18,23-25]. In this context, we also include ferroelectric precursors, which occur in the paraelectric phase and exhibit locally the structural features which define the ferroelectric phase [16-18,23-25]. Local polar structures have recently been observed by electron microscopy in the paraelectric phase of ferroelectric $\mathrm{BaTiO}_{3}$ and its solid solution with ferroelastic or incipient ferroelectric $\mathrm{SrTiO}_{3}$ with sizes of 2 to $4 \mathrm{~nm}[26,27]$. This class includes locally polar structures in relaxors (or relaxor ferroelectrics), which remain cubic down to absolute zero [28,29]. In the relaxor literature, they are generally referred to as polar nanoregions (PNRs) $[7,28,29]$. The size of PNRs ranges from several to $20 \mathrm{~nm}$ in the case of the well-known relaxor PMN [29,30].

Length scales of above-mentioned local polar structures can extend from some subnanometers to, in some cases, nearly a micron $[6,19,26,27]$. We refer to all of these various structures as polar nanostructures.

Extrinsic versus intrinsic reasonings. All effects in types (a) and (b) could include intrinsic mechanisms (i.e., characteristics of the ideal, pristine bulk effects) as well as extrinsic mechanisms, and it is not always possible to distinguish between them experimentally. Examples of extrinsic effects include defect gradients formed during high-temperature synthesis of polycrystals (ceramics) [1]. Indeed, in order to observe a macroscopic piezoelectric effect under the local polarity perspective it is necessary that the local piezoelectric tensor components $\mathrm{d}_{\mathrm{ik}}(\mathrm{r})$ do not self-compensate inside the sample; e.g., their sum over all sites has to be nonzero. This can be pictured by an "effective bias field" which prevents self-compensation, which would be expected in the thermodynamic limit and random distributions of the tensor components $[1,31,32]$. Current understanding is that this bias may come from defect gradients as an extrinsic factor formed during high-temperature synthesis of polycrystals (ceramics) [1]. The same mechanism has been proposed to occur during growth of single crystals [1]. Other mechanisms that suggest an external mechanism have also been reported [2,3,33]. However, there is also a scenario that will retain a net polarization even if the local polar entities are not subjected to an effective bias field. Such are the simulations [31] that suggest that a collection of polar entities (i.e., polar nanostructures) in materials with no external defects can also lead to a net polarization. In this case, nucleation of the first polar nanostructure biases the rest of the material, rendering it polar and piezoelectric.

Considering that mechanisms (a) and (b) raised previously regarding the effective loss of inversion symmetry leading to piezoelectricity in nominally cubic paraphases were generic and not specific and that the data are not always uniformly certain and may include real-world unavoidable defects led us to systematically measure the piezoelectric effect in nominally centrosymmetric materials. Whereas piezoelectricity is a very old effect (discovered in 1880 by the Curie brothers) and is indispensable with a vast number of technological applications [34,35], finding a new twist to it is unusual and exciting physics. We measured the resonant piezoelectric spectroscopy (RPS) $[5,10,36]$ of five unpoled ferroelectric compounds (characterized by ferroelectric domains) and their paraelectric phases (with local polar structures including ferroelectric precursors), ferroelastic $\mathrm{LaAlO}_{3}$ (with polar twin walls), four relaxor ferroelectrics (with PNRs) above their freezing temperature, incipient ferroelectrics $\mathrm{KTaO}_{3}$ and $\mathrm{SrTiO}_{3}$ with no known polar clusters at room temperature, and materials with low concentrations of defects, namely, $\mathrm{NaCl}, \mathrm{CaF}_{2}$, and silica glass. By using piezoelectric quartz single crystal as a standard, we first determine the strain generated in resonant ultrasound spectroscopy (RUS) and calibrate the piezoelectric response detected by RPS on poled ferroelectrics lead zirconate titanate $(\mathrm{PZT}-5 \mathrm{H})$ and $\mathrm{LiNbO}_{3}$, and paraelectric $\mathrm{SrTiO}_{3}$. Then, we show that nominally centrosymmetric phases of materials with local polar entities and chemical defects, like dopants, possess measurable piezoelectric effects that vary by seven orders of magnitude in comparison with poled ferroelectrics that have high piezoelectric coefficients. These values range from those comparable to that of quartz to $10 \mathrm{am} / \mathrm{V}$, which is three orders of magnitude below the detection limit of conventional piezoelectric measurements. Figure 1 is discussed in detail in this paper. In light of these results we advocate the idea that piezoelectricity is a common phenomenon in nominally centrosymmetric materials and unpoled ferroelectrics. Previous structural probes applied to the paraelectric phases suggested small deviations from crystallographic inversion symmetry even in high-quality samples (for example, Refs. [38-40]). Here we connect such structural observations with spectroscopic observation of weak piezoelectricity, an observation made possible by the extraordinary sensitivity of the advanced resonant techniques RPS and RUS. The small deviations from centrosymmetry are well reflected by piezoelectric coefficients that are as low as $0.1 \%$ to $0.001 \%$ of that of piezoelectric quartz.

\section{METHODS}

Measurements were performed on 15 compounds with 18 different samples. Five samples are ferroelectric: a $\mathrm{BaTiO}_{3}$ single crystal, a $\mathrm{BaTiO}_{3}$ ceramic, a $\mathrm{LiTaO}_{3}$ single crystal, ceramics of $(1-x) \mathrm{BaTiO}_{3}-x \mathrm{BaZrO}_{3}$ solid solution 


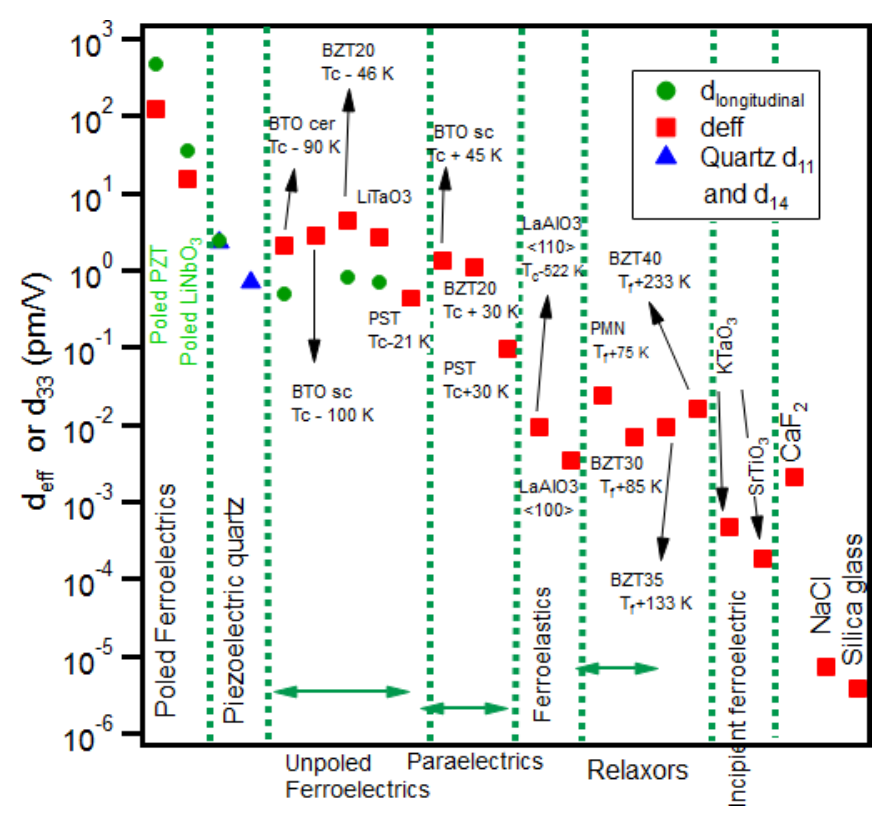

FIG. 1. Piezoelectricity in poled ferroelectrics, unpoled ferroelectrics, paraelectric phases of ferroelectrics, nominally centrosymmetric materials, and silica glass. The effective piezoelectric coefficient $\mathrm{d}_{\mathrm{eff}}$, of each sample, as determined by combined RPS and RUS measurements, corresponds to a coefficient that receives contributions from individual piezoelectric coefficients of the sample. Green circles correspond to longitudinal piezoelectric coefficients measured by a Berlincourt $\left(\mathrm{d}_{33}\right)$ meter. Triangles are piezoelectric coefficients $d_{11}$ and $d_{14}$ of quartz reported by Ref. [37].

with ferroelectric properties $(x=0.20)$. Relaxors are $(1-$ $x) \mathrm{BaTiO}_{3}-x \mathrm{BaZrO}_{3}$ with $(x=0.30,0.35,0.40)$ and PMN ceramic. Nominally nonferroelectrics are $\mathrm{LaAlO}_{3}$ single crystals ([001] and [110] oriented) in their ferroelastic phase, a single crystal of $\mathrm{KTaO}_{3}$, two $\mathrm{SrTiO}_{3}$ single crystals, quartz single crystal (X-cut), $\mathrm{NaCl}$ single crystal, $\mathrm{CaF}_{2}$, and silica glass. Ferroelectric, ferroelastic, relaxor, or isotropic behavior of each sample and characteristic temperatures are listed in Table I. $\mathrm{BaTiO}_{3}$ and $\mathrm{BaTiO}_{3}-\mathrm{BaZrO}_{3}$ ceramics were fabricated by a conventional solid-state reaction method with starting chemicals of $\mathrm{BaTiO}_{3}(99.9 \%)$ and $\mathrm{BaZrO}_{3}(99 \%)$. The calcination was performed at $1350^{\circ} \mathrm{C}$ and sintering was done at $1450{ }^{\circ} \mathrm{C}$ in air. These samples were preliminarily characterized by $\mathrm{x}$-ray diffraction. A PMN ceramic was provided by Niall Donnelly. The $\mathrm{PbSc}_{0.5} \mathrm{Ta}_{0.5} \mathrm{O}_{3}$ (PST) sample is the same as used in an earlier study [44]. A NaCl single crystal was purchased from Ted Pella Inc. while other crystals and fused silica were purchased from Hefei Kejing Materials Technology. Additionally, poled ferroelectrics (Y-36 ${ }^{\circ}$-cut) $\mathrm{LiNbO}_{3}$ single crystal and PZT-5H ceramic (not listed in Table I) were purchased from Kejing Materials Technology and Baoding Hongsheng Co. Ltd., respectively. The samples were rectangular parallelepiped platelets with surface areas ranging from 16.98 to $100 \mathrm{~mm}^{2}$ and thicknesses in the range of 0.1 to $1.17 \mathrm{~mm}$. Sample dimensions and orientations of single crystals are listed in the Supplemental Material [45].

Direct piezoelectric constant measurements were done with a quasistatic $d_{33}$ meter (IACAS, ZJ-4AN). For RUS and RPS measurements (Fig. 2), signal generation and detec- tion were achieved via an HF2LI function generator/lock-in amplifier unit (Zurich Instruments). All measurements were performed with PZT transducers with the exception of the $\mathrm{BaTiO}_{3}$ single crystal, for which $\mathrm{LiNbO}_{3}$ transducers were used, and $0.8\left(\mathrm{BaTiO}_{3}\right)-0.2\left(\mathrm{BaZrO}_{3}\right)$ in the paraelectric phase for which PIN-PMN-PT transducers were used. Each sample was mounted between two transducers along its corners, which maximize their resonance intensities. RPS and RUS measurements of each sample were performed without changing the sample position. Measurements in the paraelectric phases of $\mathrm{BaTiO}_{3}, \mathrm{BZT} 20$, and PST and the ferroelectric phase of PST were performed in a nitrogen-gascooled furnace (Sigma 10M10J or Suns Electronic Systems $\mathrm{EC} 1 \mathrm{X})$. Other measurements were done at room temperature (290-298 K). A comparison of the effective piezoelectric coefficients was made by the sum over the resonance profiles of all resonance peaks (using IGOR PRO, WAVEMETRICS INC.). For samples with high peak intensities, RUS spectra of all compounds and RPS spectra in ferroelectrics, singlecrystal quartz, and in the paraelectric phases of $\mathrm{BaTiO}_{3}$ and BZT20, the area of the spectrum was evaluated via integration, and the background was subtracted. In other materials, the total RPS area was calculated by fitting an asymmetric Lorentzian function to each resonance peak. RUS spectra were then used to scale the RPS spectra to estimate the effective piezoelectric coefficients (see Sec. III).

\section{COUPLED RPS-RUS MEASUREMENTS AS A METHOD TO MEASURE PIEZOELECTRICITY WITH HIGH RESOLUTION}

In this section, upon giving a brief discussion of the physical mechanisms behind RPS and RUS we introduce RPS-RUS measurements as a method to measure effective piezoelectric coefficients of samples with high resolution. Then, we calibrate our results by using known ferroelectrics and paraelectric $\mathrm{SrTiO}_{3}$. Other mechanisms that are allowed to contribute to the piezoelectric effect in RPS measurements were then evaluated on $\mathrm{SrTiO}_{3}$.

\section{A. Physical mechanisms behind RUS and RPS measurements}

The experimental arrangement for RUS and RPS is shown in Fig. 2(a). Both methods excite elastic standing waves (mechanical resonances) in the sample, whose frequencies are determined by the elastic moduli $[5,10,46,47]$. In RUS, an $\mathrm{AC}$ voltage is applied across the piezoelectric emitter, which vibrates through the inverse piezoelectric effect $[46,47]$. These vibrations lead to strain oscillations in the sample, which is in contact with the emitter (Fig. 2). If the frequency of oscillations corresponds to one of the natural frequencies of the sample, the oscillations become elastic standing waves (i.e., mechanical resonances). The detection is then achieved by the piezoelectric detector via the direct piezoelectric effect. In RPS, the piezoelectric emitter is not used. Instead, the AC voltage is applied across the sample. The mechanical resonances of the sample are excited if the material is macroscopically piezoelectric $[5,10,11,36,48]$. Examples in the case of single-crystal quartz, poled PZT-5H, and poled $\mathrm{LiNbO}_{3}$ are 
TABLE I. Characteristics of compounds used in this work. Freezing temperatures, $\mathrm{T}_{\mathrm{f}}$, associated with the freezing or slowing down of PNRs in relaxors, are from Refs. [41-43].

\begin{tabular}{|c|c|c|c|c|c|}
\hline $\begin{array}{l}\text { Compound } \\
\text { name }\end{array}$ & Classification & $\begin{array}{l}\text { Characteristic } \\
\text { temperature }\end{array}$ & $\begin{array}{l}\text { Compound } \\
\text { name }\end{array}$ & Classification & $\begin{array}{c}\text { Characteristic } \\
\text { temperature }\end{array}$ \\
\hline $\mathrm{BaTiO}_{3}$ & Ferroelectric & $\begin{array}{l}\mathrm{T}_{\mathrm{c}}=401 \\
\mathrm{~T}_{\mathrm{c}}(\text { ceramic })= \\
393 \mathrm{~K}\end{array}$ & $\begin{array}{l}0.6 \mathrm{BaTiO} 3- \\
0.4 \mathrm{BaZrO} 3 \\
\text { (BZT40) }\end{array}$ & Relaxor & $\mathrm{T}_{\mathrm{f}}=65 \mathrm{~K}$ \\
\hline $\mathrm{LiTaO}_{3}$ & Ferroelectric & $\mathrm{T}_{\mathrm{c}}=891 \mathrm{~K}$ & $\mathrm{LaAlO}_{3}$ & Ferrolastic & $\mathrm{T}_{\mathrm{c}}=820 \mathrm{~K}$ \\
\hline $\begin{array}{l}0.8 \mathrm{BaTiO}_{3}-0.2 \\
\mathrm{BaZrO}_{3} \\
(\mathrm{BZT} 20)\end{array}$ & Ferroelectric & $\mathrm{T}_{\mathrm{c}}=298 \mathrm{~K}$ & $\mathrm{KTaO}_{3}$ & $\begin{array}{l}\text { Incipient } \\
\text { ferroelectric }\end{array}$ & - \\
\hline $\begin{array}{l}\mathrm{PbSc}_{0.5} \mathrm{Ta}_{0.5} \mathrm{O}_{3} \\
\text { (PST) }\end{array}$ & Ferroelectric & $\mathrm{T}_{\mathrm{c}}=296 \mathrm{~K}$ & $\mathrm{SrTiO}_{3}$ & $\begin{array}{l}\text { Incipient } \\
\text { ferroelectric, } \\
\text { Ferroelastic }\end{array}$ & $\mathrm{T}_{\mathrm{c}}=105 \mathrm{~K}$ \\
\hline Quartz & Piezoelectric & $\mathrm{T}_{\mathrm{c}}=846 \mathrm{~K}$ & $\mathrm{CaF}_{2}$ & $\begin{array}{l}\text { Centrosymm } \\
\text { etric }\end{array}$ & - \\
\hline $\begin{array}{l}\mathrm{PbMg}_{1 / 3} \mathrm{Nb}_{2 / 3} \\
\mathrm{O}_{3}(\mathrm{PMN})\end{array}$ & Relaxor & $\mathrm{T}_{\mathrm{f}}=225 \mathrm{~K}$ & $\mathrm{NaCl}$ & $\begin{array}{l}\text { Centrosymm } \\
\text { etric, } \\
\text { ionic crystal }\end{array}$ & - \\
\hline $\begin{array}{l}0.7 \mathrm{BaTiO}_{3}-0.3 \\
\mathrm{BaZrO}_{3} \\
(\mathrm{BZT} 30)\end{array}$ & Relaxor & $\mathrm{T}_{\mathrm{f}}=213 \mathrm{~K}$ & Silica glass & Amorphous & - \\
\hline $\begin{array}{l}\text { 0.65BaTiO3- } \\
0.35 \mathrm{BaZrO} 3 \\
\text { (BZT35) }\end{array}$ & Relaxor & $\mathrm{T}_{\mathrm{f}}=165 \mathrm{~K}$ & & & \\
\hline
\end{tabular}

shown in Fig. 2. These resonances appear as peaks in both RPS and RUS spectra.

\section{B. How to extract the piezoelectric response from the spectra}

In RPS, because the excitation of elastic resonances requires the sample to be piezoelectric, the area of resonance peaks gives a measure of the piezoelectric effect $[8,36,49]$. The area of peaks can be calculated simply by integration or fitting an asymmetric Lorentzian function to each resonance peak (see Sec. II).

\section{Examples of earlier works on the sensitivity of RPS}

RPS was specifically designed to detect minuscule piezoelectricity that cannot be measured by conventional direct piezoelectric measurements. The latter tends to have a resolution between 0.1 and $0.01 \mathrm{pm} / \mathrm{V}$. The sensitivity of the RPS is because of the resonance condition of elastic standing waves $[5,8,10,11,36,48]$. This has previously been shown as observation of macroscopic piezoelectricity dominated by nanoscale microstructures and defects in nominally centrosymmetric materials. These include piezoelectric twin walls in the ferroelastic phase of $\mathrm{SrTiO}_{3}$ [10]. The polar (consequently piezoelectric) nature of domain walls was later demonstrated by microscopic measurements [9]. Another example is coherent defect dipoles at cryogenic temperatures in incipient ferroelectric $\mathrm{KTaO}_{3}$ [36]. In this case, defect dipoles freeze in a coherent fashion, leading to an increase in piezoelectricity with decreasing temperature. In line with these measurements, complementary measurements showed spurious ferroelectricity in the same temperature range. Finally, in relaxor ferroelectric PMN, piezoelectricity detected over a large temperature above room temperature was attributed to PNRs biased by chemically ordered regions [8]. Recent observation of macroscopic polarization at room temperature confirms the piezoelectricity in PMN [7]. The resolution of the technique was previously not explored. The lack of data on the (unexpected) piezoelectric coefficients of these centrosymmetric materials in the literature indicates that it has a higher resolution than $0.01 \mathrm{pm} / \mathrm{V}$.

\section{Measurements of strain and effective piezoelectric coefficients}

To have access to piezoelectric coefficients that are beyond the resolution of direct piezoelectric measurements, we develop a method based on RPS and RUS. This is done as follows. 

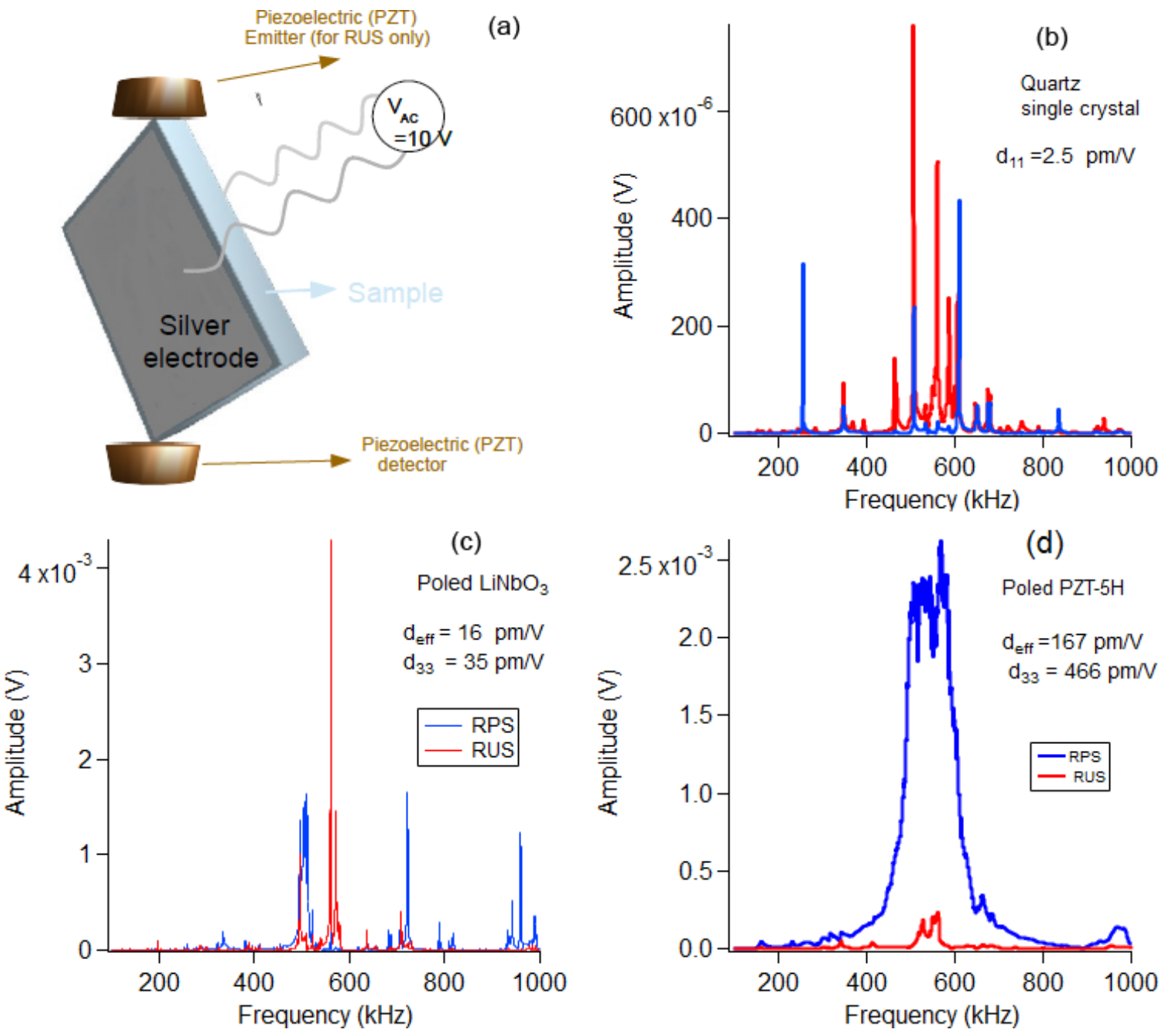

FIG. 2. Elastic resonances of single-crystal quartz, poled $\mathrm{LiNbO}_{3}$, and poled PZT-5H measured piezoelectrically (by RPS) and mechanically (by RUS). (a) Schematic of RUS and RPS measurements. The sample is lightly held between two transducers. For RPS measurements, $\mathrm{V}_{\mathrm{AC}}=1-20 \mathrm{~V}$ was applied across the sample and the resonances were detected with the piezoelectric detector. For RUS measurements, the AC voltage was applied on the emitter (instead of the sample) and the resonances were detected by using the same piezoelectric detector. RPS and RUS spectra of (b) quartz, (c) poled $\mathrm{LiNbO}_{3}$, and (d) poled PZT-5H.

\section{Quartz as standard material and its piezoelectric properties}

Piezoelectric coefficients of single-crystal quartz are $\mathrm{d}_{14}=$ $0.727 \mathrm{pm} / \mathrm{V}$ and $\mathrm{d}_{11}=2.31 \mathrm{pm} / \mathrm{V}$, as reported by Bechmann [37]. The $d_{11}$ coefficient of the sample used in this work was $\mathrm{d}_{11}=2.5 \pm 0.1 \mathrm{pm} / \mathrm{V}$ and is in line with the latter value. Note that our value is a result of direct piezoelectric measurements by a Berlincourt $\left(\mathrm{d}_{33}\right)$ meter and not by RPS and RUS measurements. Small differences between reported values of $d_{11}$ naturally occur from sample to sample [37,50].

\section{Calculation of average strain induced in RUS}

Strain induced in quartz by the application of an electric field (i.e., in an RPS experiment) can be calculated by known piezoelectric coefficients of quartz through $e_{\mathrm{RPS}}$ quartz $=\mathrm{d}_{\mathrm{av}}$ $E$, where $\mathrm{d}_{a v}$ is the average piezoelectric coefficient and $E$ is the electric field given by $V / t$ with $V$ being the applied AC voltage and $t$ the sample thickness. Here, as both longitudinal and shear coefficients $\left(\mathrm{d}_{11}\right.$ and $\left.\mathrm{d}_{14}\right)$ contribute to the generated strain, we use $d_{a v}=1 / 2\left(d_{11}+d_{14}\right)$. If the area of the RPS spectrum [RPS(peak)area] designates the strain generated by the application of the AC field and the area of the RUS spectrum corresponds to the strain in a RUS experiment $\left(e_{\mathrm{RUS}}\right)$, i.e., RUS(peak)area $\equiv e_{\mathrm{RUS}}$, comparing the two areas, one can calculate the RUS strain:

$e_{\mathrm{RUS}}=\mathrm{RUS}\left(\right.$ peak)area $\times e_{\mathrm{RPS}}($ quartz $) / \mathrm{RPS}($ peak)area.

The error in strain. The strain value depends on the piezoelectric $\left(\mathrm{d}_{14}\right.$ and $\left.\mathrm{d}_{11}\right)$ coefficients (i.e., piezoelectric anisotropy) of quartz as well as the quality and geometry of the contact between the sample and transducers. To estimate the standard deviation in RUS strain, a total of 26 RPS-RUS measurements were carried out. An example of resulting spectra is shown in Fig. 2(b). For every pair of RPS and RUS measurements, the sample was remounted to take 
into account the variations in local contact between the sample and transducers. The result is $e_{\mathrm{RUS}}=3.5 \times 10^{-7}$ with a standard deviation of $35 \%$. The calculated RUS strain spans the value of strain $\left(3 \times 10^{-7}\right)$ associated with a shear resonance generated during RUS measurements reported by Ref. [51].

\section{Measurements and calibration of piezoelectric response to calculate the effective piezoelectric coefficients $\mathrm{d}_{\mathrm{eff}}$ of samples}

This is done with a reverse procedure. Knowing the RUS strain ( $\left.e_{\mathrm{RUS}}\right)$ and comparing the areas of RPS and RUS spectra (total areas of RPS and RUS peaks) of the sample, one can calculate the strain generated in RPS, which then gives the effective piezoelectric coefficient through the equation $e_{\mathrm{RPS}}($ sample $)=\mathrm{d}_{\mathrm{eff}} E$.

The error of effective piezoelectric coefficients. Due to the range of values of the RUS strain, which is a result of piezoelectric anisotropy and quality and local geometry of contact between the sample and transducers, the effective piezoelectric coefficients determined with the approach adopted here should only be correct within an order of magnitude. Below, we test this by measuring the piezoelectric coefficients of poled ferroelectrics and paraelectric $\mathrm{SrTiO}_{3}$.

Effective piezoelectric coefficients of poled ferroelectrics. Using the approach laid out above, the effective piezoelectric coefficients of poled $\mathrm{LiNbO}_{3}$ and PZT-5H measured by RPS/RUS measurements [Figs. 2(c) and 2(d)] are $\mathrm{d}_{\text {eff }}=15$ and $167 \mathrm{pm} / \mathrm{V}$. The longitudinal piezoelectric coefficients of these samples measured by a Berlincourt $\left(\mathrm{d}_{33}\right)$ meter are $d_{\text {longitudinal }}=35 \mathrm{pm} / \mathrm{V}$ for $\mathrm{LiNbO}_{3}$ and $\mathrm{d}_{33}=466 \mathrm{pm} / \mathrm{V}$ for PZT-5H.

The electrostrictive coefficient of paraelectric $\mathrm{SrTiO}_{3}$. Because a comparison between $\mathrm{d}_{\mathrm{eff}}$ measured by RPS-RUS measurements and direct $\mathrm{d}_{33}$ measurements are not possible for values below $0.1 \mathrm{pm} / \mathrm{V}$, i.e., the resolution of our $\mathrm{d}_{33}$ meter, or below $0.01 \mathrm{pm} / \mathrm{V}$ for more sensitive $\mathrm{d}_{33}$ meters, we use a different approach to calibrate the $\mathrm{d}_{\mathrm{eff}}$ values. Instead we determine the electrostrictive coefficient of $\mathrm{SrTiO}_{3}$. This material is interesting not only on account of its archetypal status (the "Drosophila of oxide physics" as Müller once called it), but also its structural, piezoelectric, and electrostrictive properties are well documented. In addition, $\mathrm{SrTiO}_{3}$ is an apparently perfect cubic perovskite above $T_{c}=105 \mathrm{~K}$, and any elastic softening above $T_{c}$ is associated with heterogeneities. It is therefore a perfect material for the study of nanopiezoelectricity in nonpolar materials.

$\mathrm{SrTiO}_{3}$ has a very large dielectric constant $\left(\varepsilon_{r}=300\right.$ at room temperature), making it an easily polarizable material. This means that an electric field can be used to turn $\mathrm{SrTiO}_{3}$ from a nonpolar material into a polar one [52], and then measure the "field-induced piezoelectricity" as a function of electric bias. In an electrostrictive material such as $\mathrm{SrTiO}_{3}$, the strain is related to the polarization $P$ by $e=Q P^{2}$, where $Q$ is the electrostrictive coefficient. The polarization in a perfectly nonpolar crystal is the dielectrically induced polarization, $P=$ $\varepsilon_{0} \varepsilon_{r} E$, where $E$ is the electric field and $\epsilon_{0}$ is the permittivity of free space. In addition, however, there can also be a residual parasitic polarization coming from polar nanoregions, defect gradients, and so forth; the total polarization is thus $P=P_{\text {res }}+\varepsilon_{0} \varepsilon_{r} E$ with $P_{\text {res }}$ being residual polarization. The effective "induced piezoelectric" coefficient $\left(d_{\text {eff }}\right)$ is, by definition, the derivative of the strain with respect to the electric field:

$$
d_{\mathrm{eff}}=\frac{\partial e}{\partial E}=\frac{\partial e \partial P}{\partial P \partial E}=2 Q P \varepsilon_{0} \varepsilon_{r}=2 Q \varepsilon_{0} \varepsilon_{r}\left(P_{\mathrm{res}}+\varepsilon_{0} \varepsilon_{r} E\right) .
$$

In a parallel-plate capacitor, the electric field is the voltage $(V)$ divided by thickness $(t)$. Hence,

$$
d_{\mathrm{eff}}=2 Q \varepsilon_{0} \varepsilon_{r}\left(P_{\mathrm{res}}+\varepsilon_{0} \varepsilon_{r} \frac{V}{t}\right) .
$$

The above equation means that we can linearly modify the piezoelectric coefficient of an $\mathrm{SrTiO}_{3}$ single crystal by increasing the external bias voltage; the slope of the piezoelectric coefficient as a function of DC bias will be proportional to the electrostrictive coefficient and dielectric constant, while the intercept at the origin will be related to the residual macroscopic polarization of the sample. The result of this experiment, using an $\mathrm{SrTiO}_{3}$ single crystal of $0.1 \mathrm{~mm}$ thickness, is shown in Fig. 3. Notice that the induced piezoelectric coefficients are of the order of $10^{-3} \mathrm{pm} / \mathrm{V}(1 \mathrm{fm} / \mathrm{V})$ and would be hard to measure by standard techniques.

The slope of the RPS-measured piezoelectric coefficient of $\mathrm{SrTiO}_{3}$ as a function of voltage is $\sim 3.1 \mathrm{pm} /\left(\mathrm{V} \mathrm{m}{ }^{2}\right)$. From Eq. (3) and assuming $\varepsilon_{r}=300$ and $t=0.1 \mathrm{~mm}$, this slope implies an electrostrictive coefficient $Q=0.022 \mathrm{~m}^{4} / \mathrm{C}^{2}$, which is comparable to the longitudinal electrostrictive coefficient of $\mathrm{SrTiO}_{3}, Q_{33}=0.0046 \mathrm{~m}^{4} /\left(\mathrm{C} \mathrm{m}^{2}\right)$ [52]. This showcases the sensitivity of the RPS technique and its reliability, as well as offering an alternative way of calibration. Last but not least, the measured piezoelectric coefficient at zero bias $\left(\sim 2 \times 10^{-4}\right.$ $\mathrm{pm} / \mathrm{V}$ ) implies, via Eq. (3), a residual macroscopic polarization of the order of $1.7 \mathrm{C} / \mathrm{m}^{2}$. This is about $10^{5}$ times smaller than the polarization of typical ferroelectrics such as $\mathrm{BaTiO}_{3}$; conversely, if we assume that the detected residual polarization comes from coherently oriented polar clusters with a local polarization of the same order of magnitude as that of standard perovskite ferroelectrics, it means that the volume concentration of polar clusters in $\mathrm{SrTiO}_{3}$ at room temperature is of the order of $10^{-5}$ (10 parts per million). It would be difficult to observe such a small volume fraction of static polar clusters by direct means.

The limits of the RPS technique can be further tested if one considers that the forbidden piezoelectric coefficient is primarily a result of defect-gradient-induced polarization $[1,2]$. Therefore, by the application of a DC bias in the positive direction, we show that piezoelectric resonance can be suppressed and then emerge again. This means switching the direction of residual polarization by a DC bias, further illustrating the accuracy and ultrahigh sensitivity of RPS. The piezoelectric coefficient for small DC biases (120 and $210 \mathrm{mV}$ ) is calculated to be just below $10^{-5} \mathrm{pm} / \mathrm{V}$. This sets the detection limit of RPS as $10^{-5} \mathrm{pm} / \mathrm{V}$, or $10 \mathrm{am} / \mathrm{V}$.

Resolution and accuracy of the RPS method for piezoelectric measurements. Our paper demonstrates that the RPS technique can detect piezoelectric signals down to $10^{-5} \mathrm{pm} / \mathrm{V}$. Though the resolution of our technique is high, the margin of error is within an order of magnitude of the actual piezoelectric coefficient due to piezoelectric anisotropy and 
(a) Induced piezoelectricity in $\mathrm{SrTiO}_{3}$
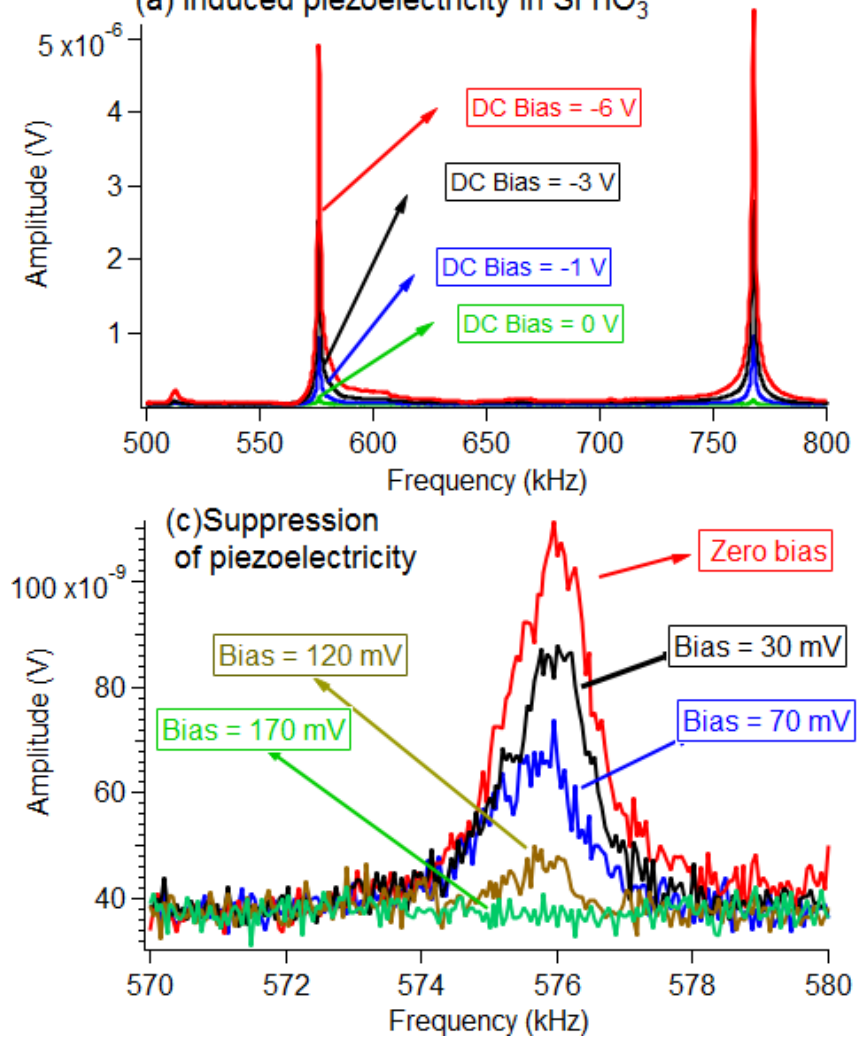

(b)

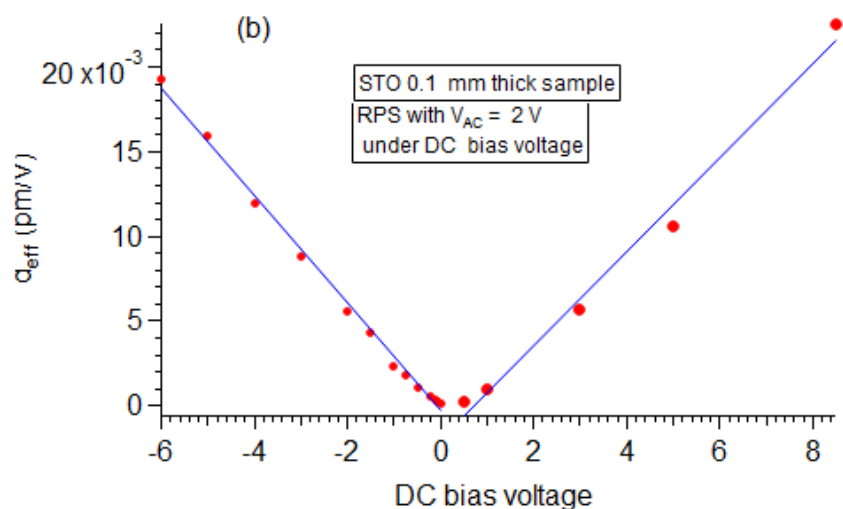

(d) Re-emergence

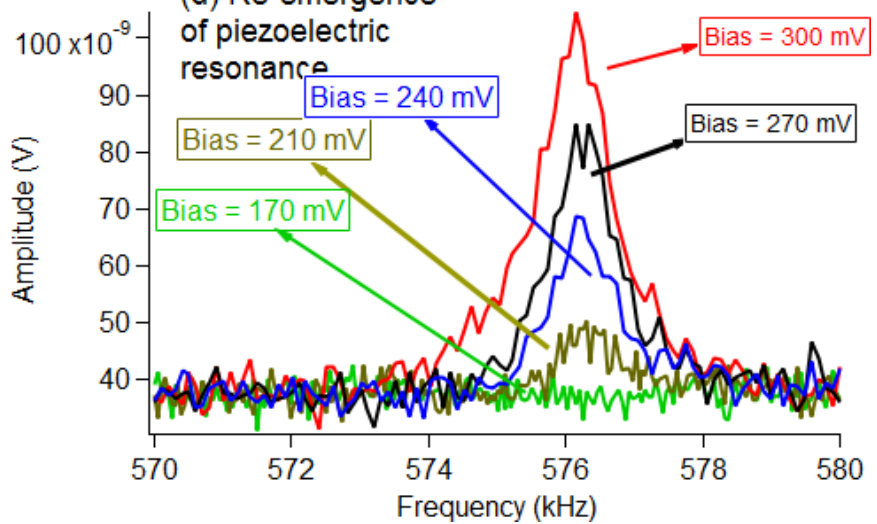

FIG. 3. Calibration of RPS signal to determine ultralow piezoelectric coefficients. (a) Induced piezoelectricity in $\mathrm{SrTiO}_{3}$ by a DC voltage bias. (b) DC bias dependence of induced piezoelectricity. (c) Suppression of piezoelectric resonance by applying a positive bias. (d) Reemergence of the resonance by applying a bias greater than $170 \mathrm{mV}$.

geometry and quality of local contact between the sample and transducers. This is well reflected in the extracted values of $\mathrm{d}_{\text {eff }}$ for poled ferroelectrics and the electrostrictive coefficient of $\mathrm{SrTiO}_{3}$, which differ from actual values by a factor of 3-5. However, this is sufficient for many scientific purposes, including the proof of existence of piezoelectricity in compounds that are nominally classified as nonpiezoelectric.

\section{E. Possible contributions of flexoelectric effect and surface piezoelectricity to RPS signals}

Here, both flexoelectricity [53] and, theoretically, surface piezoelectricity [54-57] can generate strain that can potentially be picked in our measurements. Both are very weak effects in comparison to the piezoelectric effect on bulk crystals and ceramics [55,58,59]. Nevertheless, we checked whether RPS can pick up these signals by performing sizedependent measurements on the reference material $\mathrm{SrTiO}_{3}$. The choice of $\mathrm{SrTiO}_{3}$ as a reference material stems from the fact that, in addition to being an apparently perfect paraelectric, its flexoelectric properties have been extensively investigated [60]. In fact, it is the first material for which the flexoelectric tensor was characterized [60]. Unfortunately, for other materials in this study, the information is much less complete. In any case, it would be desirable to do similar studies in other materials, although it is out of the scope of this paper. Below we explain other possible contributions to the RPS signal that we have critically examined in the case of $\mathrm{SrTiO}_{3}$

Flexoelectric effect. Bending experiments show that piezoelectricity can imitate flexoelectricity and vice versa. Although in RPS measurements we do not bend the sample, we apply a homogeneous electric field that can induce bending via inverse flexoelectricity, and this may be mistaken as a piezoelectric signal [53]. Therefore, we look at the size dependence of the RPS signal, comparing 0.1- and 1-mmthick samples (see the Supplemental Material [45]). We see that both have piezoelectric coefficients on the same order of magnitude, which indicates that the origin is truly piezoelectric and not flexoelectric; if the origin of these piezoelectric coefficients had been inverse flexoelectricity, the thinner one would have displayed an orders-of-magnitude larger signal.

Surface piezoelectricity. Surface piezoelectricity is theoretically predicted in all materials [54-57]. By symmetry, surface piezoelectricity can only manifest itself in experiments where the equivalence between opposite surfaces is broken, i.e., when there is an inhomogeneous deformation such as bending so that one surface is under tension and the other under compression. Conversely, electric fields do break the equivalence between surfaces, because a field parallel to the piezoelectricity of one surface is antiparallel 
to the piezoelectricity of the opposite surface. This should cause a material to bend. Surface piezoelectricity is therefore indistinguishable from inverse flexoelectricity (this has in fact been amply discussed in the literature), and the same size-dependent experiment that allowed us to rule out a significant role of inverse flexoelectricity also excludes a role of surface piezoelectricity. In relation to this, it has been shown that surface effects are not the dominant mechanism for the forbidden polarization detected in paraelectric phases of ferroelectrics [1]. Nevertheless, surface piezoelectricity may make some (minor) contribution to the signal detected in piezoelectric measurements. These effects are likely enhanced in the case of rough surfaces, as shown by simulations [61].

\section{PIEZOELECTRICITY IN NOMINALLY CENTROSYMMETRIC PHASES OF COMPOUNDS}

\section{A. RPS and RUS spectra of nominally centrosymmetric and bulk-centrosymmetric materials}

Examples of RPS and RUS spectra for unpoled ferroelectrics and centrosymmetric materials are shown in Fig. 4 (see Figs. S1-S3 in the Supplemental Material [45] for the spectra of other compounds and samples). Similar to piezoelectric quartz, poled $\mathrm{LiNbO}_{3}$, and poled PZT-5H, all samples show elastic resonances in RPS spectra. Due to the disallowed nature of piezoelectricity in our unpoled ferroelectrics and nominally centrosymmetric compounds, the piezoelectric coefficients must be small when self-poling effects or intentional gradients (e.g., generated by heterogeneous chemical reduction) are absent [62,63]. Biancoli et al. [1] measured the $d_{33}$ coefficient of a $\mathrm{BaTiO}_{3}$ ceramic near the ferroelectric transition temperature and found that the coefficients were on the order of $0.1-0.3 \mathrm{pC} / \mathrm{N}$ (or pm/V). In our RPS measurements, $\mathrm{BaTiO}_{3}$ as well as other ferroelectrics (Fig. 4 and the Supplemental Material [45]) show strong resonance peaks that are only slightly below RUS peak amplitudes. In other samples, while all RUS spectra contain strong resonances with amplitudes of $10^{-3}-10^{-5} \mathrm{~V}$, RPS peaks are much weaker. This defines our range of values for the unexpected piezoelectric effect.

\section{B. The magnitude of the observed piezoelectricity}

Using the approach described in Sec. III, we calculated the effective piezoelectric coefficients of other samples (Fig. 1).

In Fig. $1, \mathrm{~d}_{33}$ of poled PZT-5H ceramic and $\mathrm{LiNbO}_{3}$, piezoelectric coefficients of quartz, are also shown for the purpose of depicting the range of piezoelectric coefficients that materials can have. The overall variation of piezoelectric coefficients spans an extremely broad range of values, varying by seven orders of magnitude from 466 to $10^{-5} \mathrm{pm} / \mathrm{V}$ (or $10 \mathrm{am} / \mathrm{V})$. These results somewhat reflect the range of deviation from inversion symmetry.

Inversion symmetry is absent in poled ferroelectrics and quartz. Even unpoled ferroelectrics, although bulk centrosymmetric, can give measurable piezoelectric coefficients [1] with conventional measurements but not always [3], which also depends on the experimental resolution. Surprisingly, paraelectric phases of ferroelectrics lead to similar values that are only slightly lower (Fig. 1). This is consistent with the temperature dependence of $\mathrm{d}_{33}$ for an unpoled $\mathrm{BaTiO}_{3}$ ceramic reported by Ref. [1] which showed variations by a factor of 6 near $T_{c}$. Thus, small differences of $d_{\text {eff }}$ below and above $\mathrm{T}_{\mathrm{c}}$ in Fig. 1 do not reflect the typical depoling behavior and self-poling effects can be neglected. Taking quartz as reference, piezoelectric coefficients of centrosymmetric materials and silica glass (amorphous) range from comparable values to $\sim 10^{-3} \%$ of those of quartz $\left(\mathrm{d}_{33}=2.31 \mathrm{pm} / \mathrm{V}\right.$ and $\mathrm{d}_{14}=0.727 \mathrm{pm} / \mathrm{V}$ [37]).

\section{The role of polar nanostructures}

One should take the values of piezoelectric coefficients with caution especially for materials with local polar entities. Because the coherence between these structures is reduced on heating, one would expect the piezoelectric coefficient to also decrease with increasing temperature. As shown in Fig. 5, this is the case in the paraelectric phase of ferroelectric BZT20. This asserts earlier observations on $\mathrm{BaTiO}_{3}$ [5], PMN [8], $\mathrm{KTaO}_{3}$ [36], and $\mathrm{SrTiO}_{3}$ [10], which led to the same conclusion based on RPS measurements.

Some of the compounds investigated in this work contain polar nanostructures which have been visualized or inferred. Polar entities in $\mathrm{BaTiO}_{3}$, its solid solutions, and PST were discussed in detail [2-4,23,24,44,64-67]. These have recently been visualized in $\mathrm{BaTiO}_{3}$ [26,27] and its solid solution $\mathrm{BaTiO}_{3}-\mathrm{SrTiO}_{3}$ [26]. In the paraelectric phase of PST, striations observed in diffraction measurements [68,69] were attributed to tweed structure via Landau modeling [67] and scaling with entropy and polarization [44]. In BZT relaxors, PNRs are estimated to persist at least up to $440 \mathrm{~K}$ [41-43] while for PMN the hierarchical nanodomain structure observed at room temperature potentially exists at least up to $\sim 600 \mathrm{~K}[30,70]$. The polarity of twin walls in $\mathrm{LaAlO}_{3}$ and resulting macroscopic piezoelectricity was unambiguously demonstrated [11]. Whereas materials with known polar nanostructures presented in this work have systematically higher piezoelectric coefficients than those with no known such structures at room temperature, it remains to be determined if such nanostructures are the cause of symmetry breaking in all materials or an independent feature in some cases.

\section{Piezoelectricity in centrosymmetric materials which may contain external defects}

Among other compounds investigated here, silica glass, $\mathrm{NaCl}$, and $\mathrm{CaF}_{2}$ have no known locally polar or piezoelectric nanoclusters but contain defects [71-74]. In $\mathrm{KTaO}_{3}$ and $\mathrm{SrTiO}_{3}$ polar nanostructures only exist at cryogenic temperatures as polar clusters and polar domain walls, respectively $[9,10,36,75]$. No experimental evidence of local polarity for either sample has been reported at room temperature. Here, our results show that even cubic materials with no known polar or piezoelectric nanostructures are also piezoelectric. 

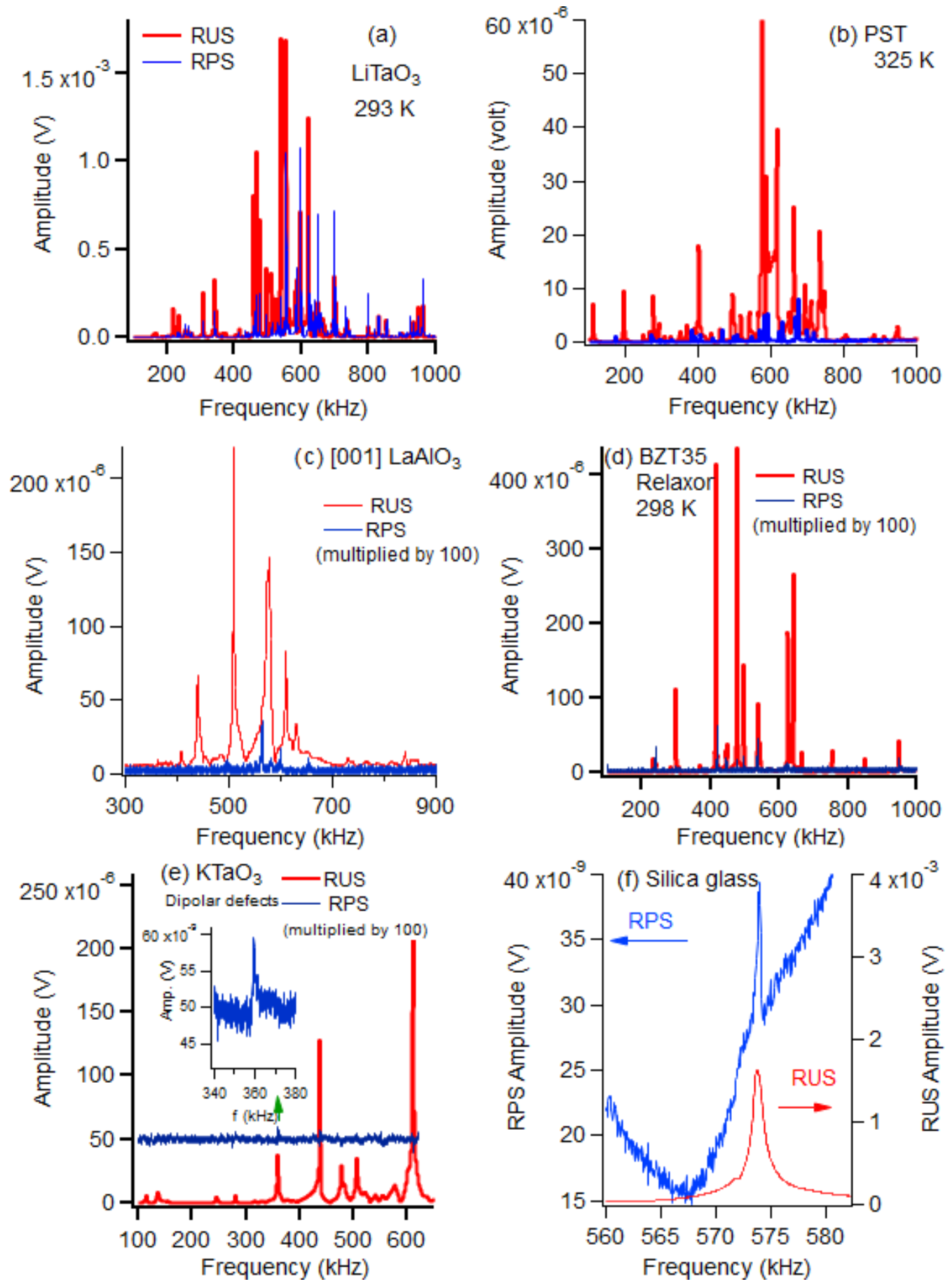

FIG. 4. Piezoelectricity in globally centrosymmetric materials and unpoled ferroelectrics which are bulk centrosymmetric due to spatial averaging of domains and grains (also see the Supplemental Material [45]). RUS spectra of all compounds show strong resonances while those detected in RPS range from $10^{-3}$ to $\sim 10^{-8} \mathrm{~V}$, demonstrating broken centrosymmetry.

\section{CURRENT UNDERSTANDING OF SPONTANEOUS ATOMIC-SCALE SYMMETRY BREAKING IN PARAPHASES}

The simplest, albeit naïve, view of a paraelectric phase according to past studies assumes that it has a net global zero dipole because each site has a zero dipole. This "nonelectric model" of paraelectricity has been often used in electronic structure calculations as it allows one to use the smallest, highest-symmetry crystallographic unit cell. Analogous approximations were common for describing paramagnets as a phase where each site has zero moment. Because it is artificial to expect a transition from finite dipoles in the low-temperature ferroelectric phase to zero dipole in the paraelectric phase, a better approximation has been the displacive model of paraelectric phases [76] that still uses the minimal unit cell but allows for finite polar displacements in a single double-well picture. However, because of the restriction 


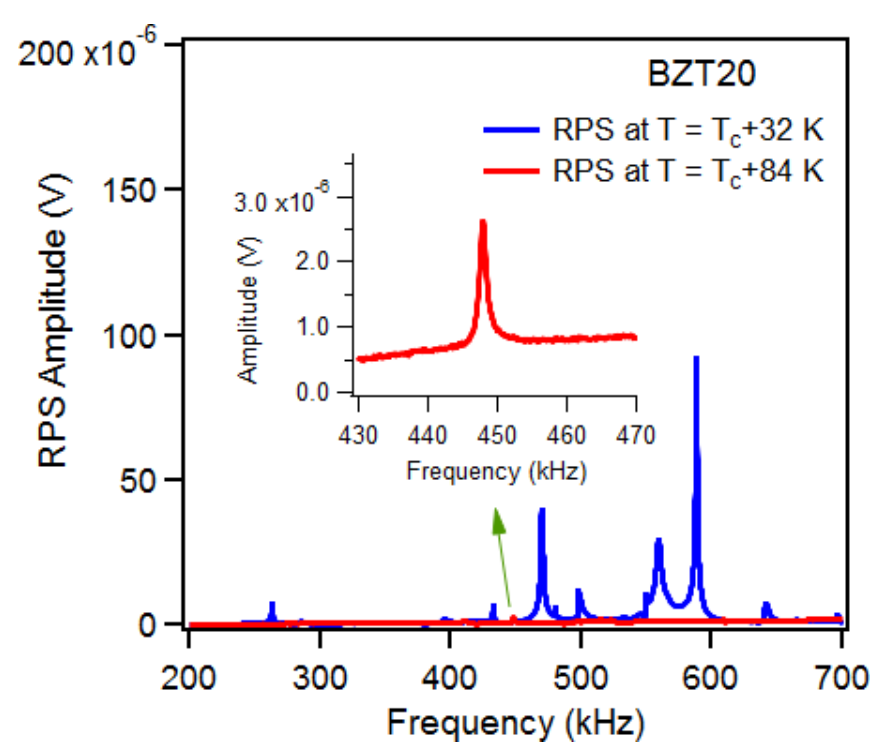

FIG. 5. Comparison of RPS spectra of ferroelectric BZT20 collected 32 and $84 \mathrm{~K}$ above the ferroelectric Curie temperature $\mathrm{T}_{\mathrm{c}}=296 \mathrm{~K}$.

to a minimal unit cell in the displacive model, all dipoles in the paraelectric phase must be aligned in tandem, leading to a long-range-ordered model for paraelectricity. In this view atoms are oscillating thermally in a single potential well around their average Wyckoff positions. Therefore, one can always apply a time average onto the observable properties. Reciprocal-space calculation of phonons is based on such a symmetry-unbroken monomorphous structure for the paraphase following the softening of the paraphonons as the transition to the lower-temperature low-symmetry phase is approached. In the alternative order-disorder model for the transitions [77], one allows in principle a larger than minimal unit cell for describing the paraphase so that disordered local polar displacements are possible in the paraelectric phase; i.e., there is a potential well with a few minima (four or eight) but the occupation numbers are variable, allowing net polarity. Phase transition occurs when occupational symmetry is broken. However, the net dipole is zero.

These concepts have been recently challenged [78]. Indeed, there are recent theoretical reasons [79-83] to believe that intrinsic mechanisms might be responsible for the formation of atomic-scale symmetry breaking in paraphases, manifesting short-range-ordered (SRO) local motifs ("polymorphous network") [79]. Such symmetry breaking was argued theoretically to lower the internal energy $\mathrm{U}_{0}$ even before thermal agitation sets in, leading to the formation of a distribution of local motifs in all paraphases. This includes paraelectric phases (where the pertinent degree of freedom is the local dipole), paramagnetic phases (where the pertinent local degree of freedom is the local magnetic moment), or paraelastic phases (where the pertinent degree of freedom can be a geometric-steric effect such as octahedral rotation). Significantly, even static minimization of the internal density functional energy of supercells constrained to maintain the global symmetry already showed a significant stabilization in forming the polymorphous network that lets different local motifs coexist in one large supercell. Naturally, as temper- ature sets in (modeled via density functional theory (DFT) molecular dynamics (MD) [80,83]), additional displacements take place. Significantly, both the minimization of the internal energy U (in DFT) and that of the free energy U-TS (via DFTMD) lead to symmetry breaking including the removal of inversion symmetry. Thus, although the average $\langle\mathrm{S}\rangle$ over local motifs $\left\{S_{i}\right\}$ has high symmetry (say, being centrosymmetric), this does not imply that all measured physical properties $\mathrm{P}$ would equal the property $\mathrm{P}(\langle\mathrm{S}\rangle)$ of the average structure (e.g., null piezoelectricity). This then allows the observation of physical effects that reflect noncentrosymmetric symmetry, including piezoelectricity. Recent first-principles calculations [79-82] considered a supercell of numerous Pm-3m unit cells, preserving the global cubic shape symmetry, but then minimizes the cell internal atomic forces in DFT. One finds in such constrained minimization of the internal energy $\mathrm{U}_{0}$ a symmetry breaking. Depending on the type of pertinent local degree of freedom, these local motifs can correspond to local octahedra rotations in cubic halide perovskites $\left(\mathrm{CsPb}_{3}\right)$ $[79,80]$, or to local magnetic moments in paramagnetic oxides $\left(\mathrm{YTiO}_{3}, \mathrm{YNiO}_{3}\right)$ [82], or to local displacements creating local dipoles in a paraelectric $\left(\mathrm{BaTiO}_{3}\right)[79,83]$. All such symmetry breakings represent short-range order and lead to macroscopic effects on the band structure, effective masses, and optical properties [79-82].

\section{CONCLUSIONS}

Conclusions and potential future work can be summarized as follows.

Macroscopic inversion symmetry breaking of cubic and bulk centrosymmetric materials. This was demonstrated to be common. We demonstrated that unpoled ferroelectric crystals and ceramics can allow significant piezoelectricity.

Internal versus external macroscopic symmetry breaking. We intentionally did not distinguish between internal [31,32,79-82] and external symmetry breaking [1,2] on the detected piezoelectricity. Although there is no experimental evidence of polar nanostructures in $\mathrm{NaCl}$, silica glass, $\mathrm{CaF}_{2}$, $\mathrm{KTaO}_{3}$, and $\mathrm{SrTiO}_{3}$ at room temperature, recent observations of macroscopic symmetry breaking in cubic materials due to internal effects [75-78] suggest that bulk symmetry breaking could occur even in the absence of defects. For example, birefringence observed in $\mathrm{CaF}_{2}$ has been proposed to stem from intrinsic effects [84]. Future experimental and theoretical work would then include the quantification of intrinsic and extrinsic contributions to the symmetry breaking and piezoelectricity in nominally cubic materials. A possible approach to help distinguish the contribution of defects in symmetry breaking would require growing high-quality cubic crystals, such as $\mathrm{NaCl}$, with known defect concentrations (some 10-1000 ppm). For examination of the local structure, transmission electron microscopy $[26,85]$ and dark field $x$-ray microscopy [13] are suitable methods. As macroscopic tools for investigation of inversion symmetry breaking, RPS, as shown in this work and earlier works $[8,10,11,36]$, and second harmonic generation [86], as shown by Refs. [87,88], could be carried out as a function of temperature on the same samples.

Applications. The possibility of new electromechanical devices based on paraelectrics exists. Piezoelectric 
coefficients in the paraelectric phases of ferroelectrics were found to be comparable to those of quartz and unpoled ferroelectrics. These results suggest that modifications of a collection of polar nanostructures may lead to new electromechanical devices. Such an attempt proved to be promising, for example, in Ref. [63], where introduction of heterogeneous chemical reduction led to $\mathrm{d}_{33}=321 \mathrm{pm} / \mathrm{V}$ in $\mathrm{Na}_{0.5} \mathrm{Bi}_{0.5} \mathrm{TiO}_{3}$ $\mathrm{BaTiO}_{3}$, which exceeds the piezoelectric coefficients of most ferroelectrics and is comparable to that of lead zirconium titanate (PZT) [89]. Moreover, because the influence of polar nanostructures on the forbidden piezoelectric effect can be seen over hundreds of degrees above ferroelectric Curie temperatures and freezing temperatures associated with relaxors, high-temperature piezoelectric applications, including energy harvesting, can be envisioned via the guided growth or synthesis of materials via chemical, stress, thermal, and electrical gradients $[34,63,90]$.

The scenario of intrinsic symmetry breaking short-range order in paraphases without defects and polar nanostructures. We know that positional local symmetry breaking such as displacements and octahedral rotations are seen by local structural probes in nominally cubic perovskites (viz., pair distribution function (PDF) [79]), whereas they often escape detection by volume-averaging techniques such as conventional x-ray diffraction. Indeed, so is local time- reversal-symmetry breaking predicted theoretically in paramagnets [79,81], even when the global magnetism (vector sum of local moments) vanishes. Similarly, we see in the current work that, whereas the global dipole in a paraelectric could be small or vanishing, symmetry-breaking calculations $[79,83]$ indicate that local dipoles need not vanish. Remarkably, the appearance of such local motifs as energy-lowering symmetry-breaking features in paraelastics, paramagnets, and paraelectrics can lead to macroscopic consequences, absent in the reference calculations lacking such local motifs.

\section{ACKNOWLEDGMENTS}

O.A. acknowledges the support of the Natural National Science Foundation of China (Grant No. 51850410520). E.K.H.S. was funded by EPSRC (Grant No. EP/P024904/1) and the EU's Horizon 2020 programme under the Marie Skłodowska-Curie Grant Agreement No. 861153. G.C. is funded by MINECO Grant No. SEV-2017-0706 and the Generalitat de Catalunya Grant No. 2017 SGR 579. The work of A.Z. at the University of Colorado at Boulder was supported by the U.S. National Science Foundation (NSF-DMR-CMMT Grant No. DMR-1724791) and the NDF DMREF program (Grant No. DMREF-1921949).
[1] A. Biancoli, C. Fancher, J. Jones, and D. Damjanovic, Breaking of macroscopic centric symmetry in paraelectric phases of ferroelectric materials and implications for flexoelectricity, Nat. Mater. 14, 224 (2015).

[2] S. Hashemizadeh, A. Biancoli, and D. Damjanovic, Symmetry breaking in hexagonal and cubic polymorphs of $\mathrm{BaTiO}_{3}$, J. Appl. Phys. 119, 094105 (2016).

[3] L. Garten and S. Trolier-McKinstry, Enhanced flexoelectricity through residual ferroelectricity in barium strontium titanate, J. Appl. Phys. 117, 094102 (2015).

[4] K. Wieczorek, A. Ziebiniska, Z. Ujma, K. Szot, M. Górny, I. Franke, J. Koperski, A. Soszyński, and K. Roleder, Electrostrictive and piezoelectric effect in $\mathrm{BaTiO}_{3}$ and $\mathrm{PbZrO}_{3}$, Ferroelectrics 336, 61 (2006).

[5] O. Aktas, M. A. Carpenter, and E. K. H. Salje, Polar precursor ordering in $\mathrm{BaTiO}_{3}$ detected by resonant piezoelectric spectroscopy, Appl. Phys. Lett. 103, 142902 (2013).

[6] G. F. Nataf, M. Guennou, J. M. Gregg, D. Meier, J. Hlinka, E. K. H. Salje, and J. Kreisel, Domain-wall engineering and topological defects in ferroelectric and ferroelastic materials, Nat. Rev. Phys. 2, 634 (2020).

[7] L. M. Riemer, K. Chu, Y. Li, H. Uršič, A. J. Bell, B. Dkhil, and D. Damjanovic, Macroscopic polarization in the nominally ergodic relaxor state of lead magnesium niobate, Appl. Phys. Lett. 117, 102901 (2020).

[8] O. Aktas and E. K. H. Salje, Macroscopic symmetry breaking and piezoelectricity in relaxor ferroelectric lead magnesium niobate, Appl. Phys. Lett. 113, 202901 (2018).

[9] Y. Frenkel, N. Haham, Y. Shperber, C. Bell, Y. Xie, Z. Chen, Y. Hikita, H. Y. Hwang, E. K. H. Salje, and B. Kalisky, Imaging and tuning polarity at $\mathrm{SrTiO}_{3}$ domain walls, Nat. Mater. 16, 1208 (2017).
[10] E. K. H. Salje, O. Aktas, M. A. Carpenter, V. V. Laguta, and J. F. Scott, Domains within Domains and Walls within Walls: Evidence for Polar Domains in Cryogenic $\mathrm{SrTiO}_{3}$, Phys. Rev. Lett. 111, 247603 (2013).

[11] H. Yokota, C. R. S. Haines, S. Matsumoto, N. Hasegawa, M. A. Carpenter, Y. Heo, A. Marin, E. K. H. Salje, and Y. Uesu, Domain wall generated polarity in ferroelastics: Results from resonance piezoelectric spectroscopy, piezoelectric force microscopy, and optical second harmonic generation measurements in $\mathrm{LaAlO}_{3}$ with twin and tweed microstructures, Phys. Rev. B 102, 104117 (2020).

[12] A. I. Frenkel, D. Ehre, V. Lyahovitskaya, L. Kanner, E. Wachtel, and I. Lubomirsky, Origin of Polarity in Amorphous $\mathrm{SrTiO}_{3}$, Phys. Rev. Lett. 99, 215502 (2007).

[13] H. Simons, A. B. Haugen, A. C. Jakobsen, S. Schmidt, F. Stöhr, M. Majkut, C. Detlefs, J. E. Daniels, D. Damjanovic, and $\mathrm{H}$. F. Poulsen, Long-range symmetry breaking in embedded ferroelectrics, Nat. Mater. 17, 814 (2018).

[14] Disorder and Strain-Induced Complexity in Functional Materials, edited by J. Siedel (Springer, Berlin, 2013).

[15] S. Salmani-Rezaie, K. Ahadi, W. M. Strickland, and S. Stemmer, Order-Disorder Ferroelectric Transition of Strained $\mathrm{SrTiO}_{3}$, Phys. Rev. Lett. 125, 087601 (2020).

[16] P. Lloveras, T. Castán, A. Planes, and A. Saxena, Precursor nanoscale textures in ferroelastic martensites, in Disorder and Strain-Induced Complexity in Functional Materials, edited by T. Kakeshita, T. Fukuda, A. Saxena, and A. Planes (Springer, Berlin, 2012), pp. 227-247.

[17] E. K. H. Salje, X. Ding, and O. Aktas, Domain glass, Phys. Status Solidi B 251, 2061 (2014).

[18] X. Ren, Strain glass and strain glass transition, in Disorder and Strain-Induced Complexity in Functional Materials, edited by 
T. Kakeshita, T. Fukuda, A. Saxena, and A. Planes (Springer, Berlin, 2012), pp. 201-225.

[19] D. D. Viehland and E. K. Salje, Domain boundary-dominated systems: Adaptive structures and functional twin boundaries, Adv. Phys. 63, 267 (2014).

[20] A. M. Bratkovsky, S. C. Marais, V. Heine, and E. K. H. Salje, The theory of fluctuations and texture embryos in structural phase transitions mediated by strain, J. Phys.: Condens. Matter 6, 3679 (1994).

[21] S. Kartha, T. Castán, J. A. Krumhansl, and J. P. Sethna, Spin-Glass Nature of Tweed Precursors in Martensitic Transformations, Phys. Rev. Lett. 67, 3630 (1991).

[22] P. Barone, D. Di Sante, and S. Picozzi, Improper origin of polar displacements at $\mathrm{CaTiO}_{3}$ and $\mathrm{CaMnO}_{3}$ twin walls, Phys. Rev. B 89, 144104 (2014).

[23] A. Bussmann-Holder, H. Beige, and G. Völkel, Precursor effects, broken local symmetry, and coexistence of order-disorder and displacive dynamics in perovskite ferroelectrics, Phys. Rev. B 79, 184111 (2009).

[24] K. Roleder, A. Bussmann-Holder, M. Górny, K. Szot, and A. Glazer, Precursor dynamics to the structural instability in $\mathrm{SrTiO}_{3}$, Phase Transit. 85, 939 (2012).

[25] A. Bussmann-Holder, K. Roleder, and J.-H. Ko, Instabilities in the ferro- and antiferroelectric lead perovskites driven by transition metal ion mass: From $\mathrm{PbTiO}_{3}$ via $\mathrm{PbZrO}_{3}$ to $\mathrm{PbHfO}_{3}$, J. Phys.: Condens. Matter 26, 275402 (2014).

[26] A. Bencan, E. Oveisi, S. Hashemizadeh, V. K. Veerapandiyan, T. Hoshina, T. Rojac, M. Deluca, G. Drazic, and D. Damjanovic, Atomic scale symmetry and polar nanoclusters in the paraelectric phase of ferroelectric materials, Nat. Commun. 12, 3509 (2021).

[27] K. Tsuda and M. Tanaka, Direct observation of the symmetry breaking of the nanometer-scale local structure in the paraelectric cubic phase of $\mathrm{BaTiO}_{3}$ using convergent-beam electron diffraction, Appl. Phys. Exp. 9, 071501 (2016).

[28] R. A. Cowley, S. N. Gvasaliya, S. G. Lushnikov, B. Roessli, and G. M. Rotaru, Relaxing with relaxors: A review of relaxor ferroelectrics, Adv. Phys. 60, 229 (2011).

[29] D. Fu, H. Taniguchi, M. Itoh, and $\mathrm{S}$. Mori, $\mathrm{Pb}\left(\mathrm{Mg}_{1 / 3} \mathrm{Nb}_{2 / 3}\right) \mathrm{O}_{3}$ (PMN) relaxor: Dipole glass or nano-domain ferroelectric? in Advances in Ferroelectrics, edited by A. Peláiz-Barranco (IntechOpen, London, 2012).

[30] M. Eremenko, V. Krayzman, B. A., H. Y. Playford, K. W. Chapman, J. C. Woicik, B. Ravel, and I. Levin, Local atomic order and hierarchical polar nanoregions in a classical relaxor ferroelectric, Nat. Commun. 10, 2728 (2019).

[31] E. K. H. Salje, S. Li, M. Stengel, P. Gumbsch, and X. Ding, Flexoelectricity and the polarity of complex ferroelastic twin patterns, Phys. Rev. B 94, 024114 (2016).

[32] A. Schiaffino and M. Stengel, Macroscopic Polarization from Antiferrodistortive Cycloids in Ferroelastic $\mathrm{SrTiO}_{3}$, Phys. Rev. Lett. 119, 137601 (2017).

[33] I. B. Bersuker, Pseudo Jahn-Teller effect in the origin of enhanced flexoelectricity, Appl. Phys. Lett. 106, 022903 (2015).

[34] M. Acosta, N. Novak, V. Rojas, S. Patel, R. Vaish, J. Koruza, G. A. Rossetti, and J. Rödel, $\mathrm{BaTiO}_{3}$-based piezoelectrics: Fundamentals, current status, and perspectives, Appl. Phys. Rev. 4, 041305 (2017).
[35] S. Trolier-McKinstry, S. Zhang, A. Bell, and X. Tan, High-performance piezoelectric crystals, ceramics, and films, Annu. Rev. Mater. Res. 48, 191 (2018).

[36] O. Aktas, S. Crossley, M. A. Carpenter, and E. K. H. Salje, Polar correlations and defect-induced ferroelectricity in cryogenic $\mathrm{KTaO}_{3}$, Phys. Rev. B 90, 165309 (2014).

[37] R. Bechmann, Elastic and piezoelectric constants of alphaquartz, Phys. Rev. 110, 1060 (1958).

[38] R. Comes, M. Lambert, and A. Guinier, The chain structure of $\mathrm{BaTiO}_{3}$ and $\mathrm{KNbO}_{3}$, Solid State Commun. 6, 715 (1968).

[39] M. S. Senn, D. A. Keen, T. C. A. Lucas, J. A. Hriljac, and A. L. Goodwin, Emergence of Long-Range Order in $\mathrm{BaTiO}_{3}$ from Local Symmetry-Breaking Distortions, Phys. Rev. Lett. 116, 207602 (2016).

[40] G. H. Kwei, S. J. L. Billinge, S.-W. Cheong, and J. G. Saxton, Pair-distribution functions of ferroelectric perovskites: Direct observation of structural ground states, Ferroelectrics 164, 57 (1995).

[41] T. Maiti, R. Guo, and A. S. Bhalla, Structure-property phase diagram of $\mathrm{BaZr}_{x} \mathrm{Ti}_{1-x} \mathrm{O}_{3}$, J. Am. Ceram. Soc. 91, 1769 (2008).

[42] T. Maiti, R. Guo, and A. S. Bhalla, The evolution of relaxor behavior in Ti4+ doped $\mathrm{BaZrO}_{3}$ ceramics, J. Appl. Phys. 100, 114109 (2006).

[43] V. V. Shvartsman, J. Zhai, and W. Kleemann, The dielectric relaxation in solid solutions $\mathrm{BaZr}_{\mathrm{x}} \mathrm{Ti}_{1-\mathrm{x}} \mathrm{O}_{3}$, Ferroelectrics 379, 77 (2009).

[44] G. Linyu, F. Romero, V. Franco, J.-M. Martín-Olalla, M. Gallardo, E. H. Salje, Y. Zhou, and O. Aktas, Correlations between elastic, calorimetric, and polar properties of ferroelectric $\mathrm{PbSc}_{0.5} \mathrm{Ta}_{0.5} \mathrm{O}_{3}$ (PST), Appl. Phys. Lett. 115, 161904 (2019).

[45] See Supplemental Material at http://link.aps.org/supplemental/ 10.1103/PhysRevResearch.3.043221 for dimensions of all samples, orientations of single crystals, and RPS and RUS spectra of samples not shown in the paper.

[46] A. Migliori and J. Sarrao, Resonant Ultrasound Spectroscopy: Applications to Physics, Materials Measurements, and Nondestructive Evaluation (Wiley, New York, 1997).

[47] M. A. Carpenter, Static and dynamic strain coupling behaviour of ferroic and multiferroic perovskites from resonant ultrasound spectroscopy, J. Phys.: Condens. Matter 27, 263201 (2015).

[48] J. Aufort, O. Aktas, M. A. Carpenter, and E. K. Salje, Effect of pores and grain size on the elastic and piezoelectric properties of quartz-based materials, Am. Mineral. 100, 1165 (2015).

[49] O. Aktas, J. R. Duclère, S. Quignon, G. Trolliard, and E. K. H. Salje, Polarity of modulated $\mathrm{Na}_{0.5} \mathrm{Bi}_{0.5} \mathrm{TiO}_{3}$ and its slow structural relaxation, Appl. Phys. Lett. 113, 032901 (2018).

[50] V. E. Bottom, Measurement of the piezoelectric coefficient of quartz using the Fabry-Pérot dilatometer, J. Appl. Phys. 41, 3941 (1970).

[51] H. Ogi, M. Fukunaga, M. Hirao, and H. Ledbetter, Elastic constants, internal friction, and piezoelectric coefficient of $\alpha-\mathrm{TeO}_{2}$, Phys. Rev. B 69, 024104 (2004).

[52] B. Khanbabaee, E. Mehner, C. Richter, J. Hanzig, M. Zschornak, U. Pietsch, H. Stöcker, T. Leisegang, D. C. Meyer, and S. Gorfman, Large piezoelectricity in electric-field modified single crystals of $\mathrm{SrTiO}_{3}$, Appl. Phys. Lett. 109, 222901 (2016).

[53] A. Abdollahi, N. Domingo, I. Arias, and G. Catalan, Converse flexoelectricity yields large piezoresponse force microscopy 
signals in non-piezoelectric materials, Nat. Commun. 10, 1266 (2019).

[54] J. Hong and D. Vanderbilt, First-principles theory of frozen-ion flexoelectricity, Phys. Rev. B 84, 180101(R) (2011).

[55] A. K. Tagantsev and A. S. Yurkov, Flexoelectric effect in finite samples, J. Appl. Phys. 112, 044103 (2012).

[56] M. Stengel, Microscopic response to inhomogeneous deformations in curvilinear coordinates, Nat. Commun. 4, 2693 (2013).

[57] C. Gattinoni, N. Strkalj, R. Härdi, M. Fiebig, M. Trassin, and N. A. Spaldin, Interface and surface stabilization of the polarization in ferroelectric thin films, Proc. Natl. Acad. Sci. USA 117, 28589 (2020).

[58] E. V. Bursian and O. I. Zaikovskii, Changes in the curvature of a ferroelectric film due to polarization, Sov. Phys. Solid State 10, 1121 (1968).

[59] P. Zubko, G. Catalan, and A. K. Tagantsev, Flexoelectric effect in solids, Annu. Rev. Mater. Res. 43, 387 (2013).

[60] P. Zubko, G. Catalan, P. R. L. Welche, A. Buckley, and J. F. Scott, Strain-Gradient-Induced Polarization in $\mathrm{SrTiO}_{3}$ Single Crystals, Phys. Rev. Lett. 99, 167601 (2007).

[61] G. Lu, S. Li, X. Ding, and E. Salje, Piezoelectricity and electrostriction in ferroelastic materials with polar twin boundaries and domain junctions, Appl. Phys. Lett. 114, 202901 (2019).

[62] C. He, Z. Wang, X. Li, X. Yang, X. Long, and Z.-G. Ye, Self-polarized high piezoelectricity and its memory effect in ferroelectric single crystals, Acta Mater. 125, 498 (2017).

[63] W. Zhou, P. Chen, Q. Pan, X. Zhang, and B. Chu, Lead-free metamaterials with enormous apparent piezoelectric response, Adv. Mater. 27, 6349 (2015).

[64] E. Dul'kin, J. Petzelt, S. Kamba, E. Mojaev, and M. Roth, Relaxor-like behavior of $\mathrm{BaTiO}_{3}$ crystals from acoustic emission study, Appl. Phys. Lett. 97, 032903 (2010).

[65] A. Bussmann-Holder, J.-H. Ko, A. Majchrowski, M. Górny, and K. Roleder, Precursor dynamics, incipient ferroelectricity and huge anharmonicity in antiferroelectric lead zirconate $\mathrm{PbZrO}_{3}$, J. Phys.: Condens. Matter 25, 212202 (2013).

[66] B. Mihailova, B. Maier, C. Paulmann, T. Malcherek, J. Ihringer, M. Gospodinov, R. Stosch, B. Güttler, and U. Bismayer, High-temperature structural transformations in the relaxor ferroelectrics $\mathrm{PbSc}_{0.5} \mathrm{Ta}_{0.5} \mathrm{O}_{3}$ and $\mathrm{Pb}_{0.78} \mathrm{Ba}_{0.22} \mathrm{Sc}_{0.5} \mathrm{Ta}_{0.5} \mathrm{O}_{0.5}$, Phys. Rev. B 77, 174106 (2008).

[67] O. Aktas, E. K. H. Salje, S. Crossley, G. I. Lampronti, R. W. Whatmore, N. D. Mathur, and M. A. Carpenter, Ferroelectric precursor behavior in $\mathrm{PbSc}_{0.5} \mathrm{Ta}_{0.5} \mathrm{O}_{3}$ detected by field-induced resonant piezoelectric spectroscopy, Phys. Rev. B 88, 174112 (2013).

[68] K. Z. Baba-Kishi and D. J. Barber, Transmission electron microscope studies of phase transitions in single crystals and ceramics of ferroelectric $\mathrm{Pb}\left(\mathrm{Sc}_{1 / 2} \mathrm{Ta}_{1 / 2}\right) \mathrm{O}_{3}$, J. Appl. Cryst. 23, 43 (1990).

[69] K. Z. Baba-Kishi and M. Pasciak, An electron diffraction and Monte Carlo simulation study of an incommensurate antiferroelectric state in the relaxor ferroelectric $\mathrm{Pb}_{2} \mathrm{ScTaO}_{6}$, J. Appl. Cryst. 43, 140 (2010).

[70] G. Burns and B. Scott, Index of refraction in 'dirty' displacive ferroelectrics, Solid State Commun. 13, 423 (1973).

[71] F. T. Rabouw, N. M. B. Cogan, A. C. Berends, W. v. d. Stam, D. Vanmaekelbergh, A. F. Koenderink, T. D. Krauss, and
C. d. M. Donega, Non-blinking single-photon emitters in silica, Sci. Rep. 6, 21187 (2016).

[72] A. Silin and L. Skuja, Intrinsic defects in fused silica, J. NonCryst. Solids 71, 443 (1985).

[73] L. Skuja, M. Hirano, H. Hosono, and K. Kajihara, Defects in oxide glasses, Phys. Status Solidi C 2, 15 (2005).

[74] A. Burow, M. Sierka, J. Döbler, and J. Sauer, Point defects in $\mathrm{CaF}_{2}$ and $\mathrm{CeO}_{2}$ investigated by the periodic electrostatic embedded cluster method, J. Chem. Phys. 130, 174710 (2009).

[75] H. Uwe, K. B. Lyons, H. L. Carter, and P. A. Fleury, Ferroelectric microregions and Raman scattering in $\mathrm{KTaO}_{3}$, Phys. Rev. $\mathrm{B}$ 33, 6436 (1986).

[76] W. J. Merz, The electric and optical behavior of $\mathrm{BaTiO}_{3}$ singledomain crystals, Phys. Rev. 76, 1221 (1949).

[77] I. Bersuker, On the origin of ferroelectricity in perovskite-type crystals, Phys. Lett. 20, 589 (1966).

[78] J. Hlinka, T. Ostapchuk, D. Nuzhnyy, J. Petzelt, P. Kuzel, C. Kadlec, P. Vanek, I. Ponomareva, and L. Bellaiche, Coexistence of the Phonon and Relaxation Soft Modes in the Terahertz Dielectric Response of Tetragonal $\mathrm{BaTiO}_{3}$, Phys. Rev. Lett. 101, 167402 (2008).

[79] X.-G. Zhao, G. M. Dalpian, Z. Wang, and A. Zunger, Polymorphous nature of cubic halide perovskites, Phys. Rev. B 101, 155137 (2020).

[80] X.-G. Zhao, Z. Wang, O. I. Malyi, and A. Zunger, The effects of static local distortions vs. dynamic thermal motions on the stability and band gaps of cubic oxide and halide perovskites, Mater. Today 49, 107 (2021).

[81] Z. Wang, O. I. Malyi, X. Zhao, and A. Zunger, Mass enhancement in $3 d$ and $s-p$ perovskites from symmetry breaking, Phys. Rev. B 103, 165110 (2021).

[82] J. Varignon, M. Bibes, and A. Zunger, Origin of band gaps in $3 d$ perovskite oxides, Nat. Commun. 10, 1658 (2019).

[83] X.-G. Zhao, O. I. Malyi, S. J. L. Billinge, and A. Zunger, Intrinsic local symmetry-breaking in nominally cubic paraelectric $\mathrm{BaTiO}_{3}$, arXiv:2106.05231.

[84] J. H. Burnett, Z. H. Levine, and E. L. Shirley, Intrinsic birefringence in calcium fluoride and barium fluoride, Phys. Rev. B 64, 241102(R) (2001).

[85] S. Van Aert, S. Turner, R. Delville, D. Schryvers, G. Van Tendeloo, and E. K. H. Salje, Direct observation of ferrielectricity at ferroelastic domain boundaries in $\mathrm{CaTiO}_{3}$ by electron microscopy, Adv. Mater. 24, 523 (2012).

[86] M. Fiebig, V. V. Pavlov, and R. V. Pisarev, Second-harmonic generation as a tool for studying electronic and magnetic structures of crystals: Review, J. Opt. Soc. Am. B 22, 96 (2005).

[87] G. Fox, J. Yamamoto, D. V. Miller, L. E. Cross, and S. K. Kurtz, Thermal hysteresis of optical second harmonic in paraelectric $\mathrm{BaTiO}_{3}$, Mater. Lett. 9, 284 (1990).

[88] A. M. Pugachev, V. I. Kovalevskii, N. V. Surovtsev, S. Kojima, S. A. Prosandeev, I. P. Raevski, and S. I. Raevskaya, Broken Local Symmetry in Paraelectric $\mathrm{BaTiO}_{3}$ Proved by Second Harmonic Generation, Phys. Rev. Lett. 108, 247601 (2012).

[89] J. Gao, D. Xue, W. Liu, C. Zhou, and X. Ren, Recent progress on BaTiO3-based piezoelectric ceramics for actuator applications, Actuators 6, 24 (2017).

[90] K. Gerace, J. Mauro, and C. Randall, Piezoelectric glassceramics: Crystal chemistry, orientation mechanisms, and emerging applications, J. Am. Ceram. Soc. 104, 1915 (2021). 